hfarmer@ccc.edu 


\title{
Unification of Fundamental Physics Using Lagrangian-Euclidian Space, Where GR $=$ QFT.
}

\author{
Hontas Farmer 0000-0003-1510-7786 \\ ${ }^{1}$ College of DuPage, Divison of Mathematics and Natural Sciences , 425 Fawell Blvd, Glen \\ Ellyn, Illinois, United States of America \\ ${ }^{1}$ City Colleges of Chicago-Wilbur Wright College, Department of Natural Science, 226 W. \\ Jackson St, Chicago, Illinois, United States of America \\ ${ }^{1}$ Triton College, Department of Science, 2000 N Fifth Ave, River Grove, Illinois, United \\ States of America
}

April 11, 2018

\begin{abstract}
Suppose we were to treat the Lagrangians of the theories we wish to unite as coordinates in a space of Lagrangians. In this space the Lagrangians for general relativity (Einstein-Hilbert), the standard model, and the dark sector act as coordinate axes. From here a functional derivative equation is set up for a functional, $\mathrm{T}$, which gives as a result another functional, U, which denotes the Lagrangian for our universe. From there the action $(S)$ due to $U$ is computed and shown to converge even at infinite energy. Finally with $S$ a generating functional for all possible interactions is computed and a one inch equation of everything is $\operatorname{given}\left(Z=e^{i S}\right)$. It is the assertion of this paper that with the generating functional detailed herein one may compute any physical quantity of interest including gravitational and dark sector corrections and arrive at a finite result. Specifically graviton-graviton and graviton photon interactions are shown to result in finite observable quantities. It is noted that this is a fully worked out model where GR and QFT are treated on an equal footing by relativizing QFT, or put simply GR=QFT.
\end{abstract}

\section{Introduction}

The fundamental question to ask is why our universe is so well described by at least three Lagrangians, which may not be simply unified, and which derive from very different looking physical foundations? These Lagrangians are the standard model of particle physics, the Einstein-Hilbert Lagrangian and the Lagrangian for the dark sector. There is one widely used and well studied effort at unification M-Theory. However, M-Theory is based on SUSY and can't work without it. The Large Hadron Colliders two big collaborations have not found signs of any particles predicted by the modified super symmetric standard models which are the low energy states of string theory and hence M-Theory (ATLAS Collaboration, 2017; Farmer, 2017; Search for supersymmetry using hadronic top quark tagging in $13 \mathrm{TeV}$ pp collisions, 2017). New ideas are here needed, so here is a new idea.

Rather than looking for an answer that has a certain anticipated form, that of a SUSY string or M theory, I propose a change of focus to answering three fundamental questions. What is the underlying reason that our universe has the Lagrangian that it does? Can we find a simpler more fundamental representation of known physics? Can we at least quantify the effect that dark sector physics could have on standard model particles and gravity? This work builds on the principles of relativization previously published in articles such as (and, 2014a, 2015, 2014b, b,a; Farmer, 2017) 


\section{A New View Towards Unification}

Unification in particle physics traditionally means that as we increase the energy scale in our experiments different forces begin to look the same. This worked for the electro-weak and strong force but does not work for gravity. We don't know if this will work for dark matter or dark energy which are most of the universe but we can safely assume that it will. The form of unification proposed in this paper is more akin to the unification of electricity with magnetism in the form of Maxwell's equations.

There are three fundamental Lagrangians each of which corresponds to a dimension in an otherwise standard Euclidean three-space, $(L)$. One dimension gives the standard model of particle physics $\left(\mathcal{L}_{S M}\right)$, another the Einstein-Hilbert Lagrangian $\left(L_{E H}\right)$ and the as yet unknown Lagrangian for the dark sector $(\mathcal{O})$ (for other). This space of Lagrangians has all the usual features closure under scalar multiplication dot product etc. Furthermore, this space supports calculus with the functional derivative. A vector in this space gives the Lagrangian to describe the physics of a particular universe, equation 1.

$$
U(\alpha, \beta, \omega)=\alpha \mathcal{L}_{S M}+\beta L_{E H}+\omega \mathcal{O}
$$

Each one of $(L),(\mathcal{L})$, and $(\mathcal{O})$ are as independent as x y and z coordinates. Only $(\mathcal{L})$ for the standard model of particle needs to be a quantum field theory. We can treat Einstein-Hilbert Lagrangian $(L)$ as such a theory. Will the infinities cancel out? The answer is yes, and I show how below (equation 5) but to understand the result read on.

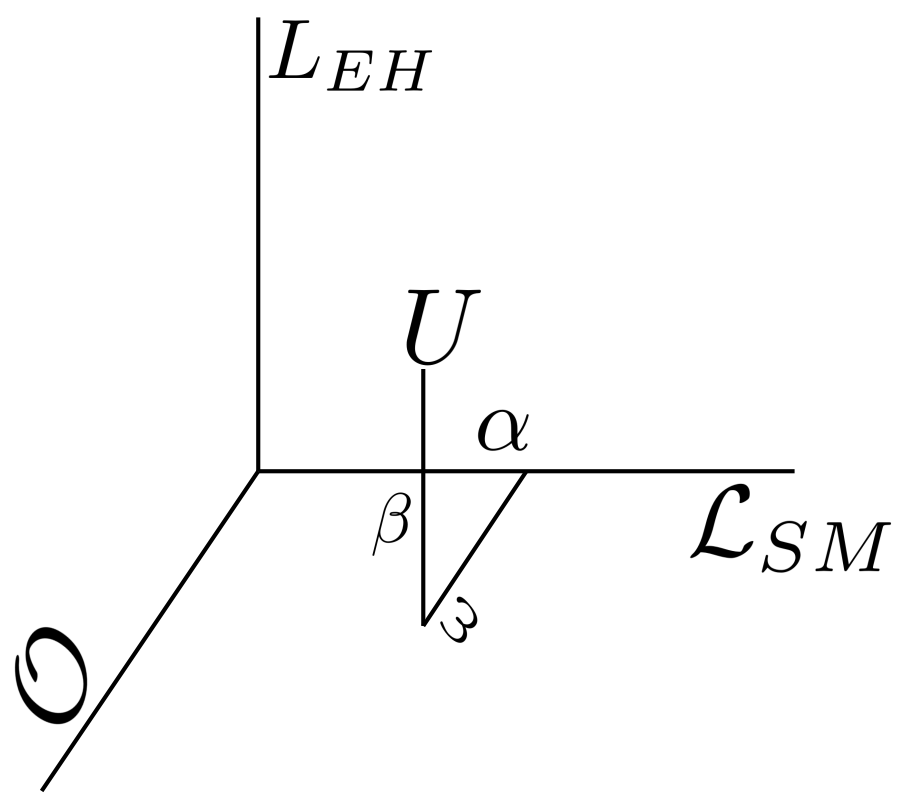

Figure 1: The space of physically meaningful Lagrangians $(L)$ is three dimensional, Euclidean, and supports calculus. 
The algebra to show that any such combination of Lagrangians is also a Lagrangian and that they form a vector space of Lagrangians is trivial.

\section{Deriving the Lagrangian for Our Universe}

We know that all Lagrangian will have to obey the principle of least action stated in the most general form possible.

$$
\delta \int U(\alpha, \beta, \omega)=0
$$

All Lagrangians must satisfy this principle in addition to matching all the observed physical data. So one may consider a space of Lagrangians $(L)$ in which all the Lagrangians differ from those in our universe by a scalar parameter.

If we define a gradient on this space using functional differentiation as follows.

$$
\nabla \equiv \frac{\delta}{\delta \mathcal{L}}+\frac{\delta}{\delta L}+\frac{\delta}{\delta \mathcal{O}}
$$

Using this derivative one may define a differential equation for a functional on $(L)$ which can give various universes.

$$
\nabla T=l(\alpha, \beta, \omega) T
$$

is a generic Lagrangian $\mathrm{T}$ is a functional from which Lagrangians can be derived.

To get a well defined first order partial differential equation we need one boundary condition or one initial condition. For this differential equation the derivative must converge uniformly to the Lagrangian of a particular universe as the Lagrangian goes to zero and as $\mathrm{T}$ goes to one. For our universe this means the solution must approach that of our universe in the low energy limit. To give finite results it should also be a mathematically well behaved functional on $(L)$. The simple anzats that will satisfy this for any given universe is equation 3 .

$$
T=e^{\beta L(\alpha \mathcal{L}-\omega \mathcal{O})}
$$

$$
\nabla T=\nabla e^{\beta L(\alpha \mathcal{L}-\omega \mathcal{O})}=\nabla(\beta L(\alpha \mathcal{L}-\omega \mathcal{O})) e^{\beta L(\alpha \mathcal{L}-\omega \mathcal{O})} \rightarrow U(\alpha, \beta, \omega)
$$


For our universe let $(\beta=\alpha=1),(\omega \ll 1)$

$$
U=(L+\mathcal{L}-\omega(\mathcal{O}+L)) e^{L(\mathcal{L}-\omega \mathcal{O})}
$$

The value of the exponential functional approaches one as the value of the Lagrangians, which are it's arguments, approach zero. Therefore, at low energies the exact physics we have observed will be seen.

If one chooses to Taylor expand $\mathrm{U}$ in equation 4 they will find an infinite progression of corrective terms. Taking those steps does not make the math that follows any easier and so showing it will be omitted from this paper.

\section{Generating Functional for Field Interactions.}

$$
Z=e^{\imath \int d^{4} x U}
$$

Having the generating function for the theory completely defines how its operators are related, in quantum field theory, but this is NOT a quantum field theory in the truest sense of the word. Each Lagrangian $L, \mathcal{L}$, and $\mathcal{O}$, is assumed to have been written in a form which has the following features, it is invariant under all space-time diffeomorphisms and the units have been canceled out perhaps by dividing by the Planck energy. Likewise the units on the actions will have been canceled out as well.

The claim of equation 5 needs to be verified experimentally. In this section I will show that the functional integrals in equation 5 will converge and that for low energies they will give the same action, and interactions that we observe at the energies we have been able to prove so far. Furthermore, I will show some of the higher energy predictions made and which may soon be testable.

$$
\int d^{4} x U=\int d^{4} x(L+\mathcal{L}-\omega(\mathcal{O}+L)) e^{L(\mathcal{L}-\omega \mathcal{O})}
$$

To simplify this expression I will integrate by parts and make use of the fact that the action of the standard model, $\mathcal{S}$, and the Einstein-Hilbert action, s, are well known. The action of the dark sector is not known but never the less the integral of it's Lagrangian will by definition be a Lagrangian, S. Carrying out this integration is not complicated given we know parts of the integral already. We will call the value of this integral $S$ for the action due to the unifiedLagrangian $\mathrm{U}$.

$$
S=\int d^{4} x U=(s+\mathcal{S}-\omega(\mathbb{S}+s)) e^{L(\mathcal{L}-\omega \mathcal{O})}
$$


Clearly if the Lagrangians go to infinity the actions also go to infinity. The relative negative sign between them ensures those infinities cancel out. The value of the exponential will approach one due to the infinities canceling to zero. Now the partition which describes the whole universe with all interactions and corrections and gives finite results can be written down as equation 6 .

$$
Z=e^{\imath(s+\mathcal{S}-\omega(\mathbb{S}+s)) e^{L(\mathcal{L}-\omega \mathcal{O})}}=e^{\imath S}
$$

With equation 6 in hand one may compute the correlation functions for any desired interactions to any desired degree of precision. It is even possible to constrain the unknown effects of dark sector interactions on standard model particles in the confines of this framework.

As a demonstration of this I will derive the Graviton Graviton crossection and show that the corrections converge to a finite quantity.

\subsection{Graviton-Graviton Correlation Function}

The major challenge in quantum gravity is to come up with a formulation which does not contain ultraviolet divergences. To demonstrate that this model does not have the UV divergence problem we now derive the correlation function from the generating functional formalism for graviton graviton interaction. Many steps will be skipped, and detailed calculations are in the supplemental material for this paper.

$$
G\left(R R^{\prime}\right)=\left.Z[J]^{-1}\left(-i \frac{\delta}{\delta J}\right)\left(-i \frac{\delta}{\delta J^{\prime}}\right) Z[J]\right|_{J=0}
$$

$$
=\left.Z[J]^{-1}\left(-i i R R^{\prime}\right) Z[J]\right|_{J=0}=e^{-\imath S} R R^{\prime} e^{\imath S}
$$

Therefore the correlation fuction

$$
G\left(R R^{\prime}\right)=R R^{\prime}
$$

Therefore when one graviton interacts with another graviton finite input gives finite output. Every term in the taylor series will have a corresponding counter term in this model. Therefore, the UV divergence problem is solved. 


\subsection{Graviton- Electromagnetic interactions.}

Another interesting cross section from a practical application standpoint would be the interaction of gravitons with electromagnetic waves and charged particles. All of our technology is based on our understanding of quantum electrodynamics and its classical limits, Maxwell's equations. If we can understand fully how gravity can interact with electromagnetism then we could, in time, develop practical technologies.

With the generating functional worked out, and the lack of UV divergence proven, it is trivial to show that the graviton-electromagnetic correlation function will be given as follows

$$
G(\bar{\psi}, \psi, A, R)=\left.Z[J]^{-1} \bar{\psi} \gamma \psi A R Z[J]\right|_{J=0}=\bar{\psi} \gamma \psi A R
$$

This can be interpreted quite simply, the correlation between electromagnetism and gravitation is linear. If this model is correct, then modifying gravity to engineer the local space-time manifold is a matter of manipulating the electro-magnetic field, and nothing dramatic will occur. $\mathrm{R}$ will change as the $\mathrm{E}$ and $\mathrm{M}$ field does but there is not a run away divergence as the previous calculation shows.

\section{Discussion}

Does this paper answer the fundamental questions listed in the introduction 1? Yes.

The underlying reason that our universe has the Lagrangian $U$ (equation 4 ) is because it is the only one that satisfies the differential equation for the function $T$ (equation 2) on the space of Lagrangians $L$ given the boundary conditions for our universe. One can then ask why our universe has the boundary conditions, why are the fundamental constants what they are, and so forth. They are what they are fundamentally because they lead to an equation $U$ that satisfies the boundary conditions of our universe. If they were any different then $\mathrm{U}$ would describe a different universe. To talk of other universes is as of right now a very popular speculation, but speculation none the less. That said, the space of Lagrangians $L$ contains as many points as a 3D euclidean space can this theory does not rule out other possibilities. Instead it constrains us to just one of those Lagrangians (equation 4).

A simpler more fundamental representation of the physics embodied in the Lagrangian $U$ is arguably given by any one of three equations in this paper. The equation giving the $\mathrm{T}$ function for our universe (equation3) is a candidate but it is simply a tool used to derive the Lagrangian U. A better answer is furnished by the generating functional Z (equation 6).

The generating functional facilitates easy computation of correlation functions between fields by way of simple functional derivatives. Using that formalism it was shown that UV divgerence is not an issue in this model for graviton-graviton, and graviton-electromagnetic field calculations.

Is this paper an example of what past publications have called a relativized model or where GR=QFT? In relativized Quantum Field Theory QFT is modified to make it obey the principles of General Relativity. The logic being that since QFT is the result of modifying quantum mechanics so that it is compatible with Special Relativity, we simply modifiy it a bit more. GR=QFT states put simply that GR is equally fundamental to QFT and that without modification one can work with both of them. The literature on this is a bit lacking in details on how to do it, for instance (Susskind, 2017). This model could also be interpreted in that manner, with QFT techniques applied to calculating quantities in GR and treating Ricci curvature as a field operator. 


\section{Conclusion}

This paper posed the question why is our universe is so well described by at least three Lagrangians, which may not be simply unified, and which derive from very different looking physical foundations? I postulated a euclidean space of Lagrangians $L$ in which the Lagrangians for the standard model $\mathcal{L}$ the Einstein-Hilbert Lagrangian $L$ and the action of the dark sector $\mathcal{O}$ (even though the details of this last one are unknown) are coordinates. From that assumption a differential equation for a functional $T$ on the space $L$ was derived. The result of that equation with the right boundary conditions is the Lagrangian for our universe $U$. With $U$ I defined the partition function $Z$. The action integral $S$ was computed and found to converge at all energies. Therefore the generating functional $Z$ can be used to compute physically meaningful quantities.

From the generating functional $\mathrm{Z}$ the results of any interaction between fields may be computed by taking functional derivatives. Equation 6 also makes manifestly obvious that there can be no infinitely energetic interactions in this universe. Equation 6 can be written as what as a simple elegant "one inch equation". Equation embodies all known physics, and allows calculations to constrain the effects of all as of yet unknown physics of the dark sector, and does so without any divergences, singularities, or infinities.

$$
Z=e^{\imath S}
$$

This theory is not simply a quantum field theory although it builds on it. This is not general relativity although it complies with the constraints of that theory. This is a new type of theory. Let us call it Lagrangian-Euclidean Space Theory (LEST), because the one postulate of this theory is that there exist a three dimensional Euclidean space of Lagrangian's in which all of these functionals exist. 


\section{References}

Hontas Farmer and. Gravity Mediated Interaction Cross Sections Without Ultraviolet Divergence., a. URL https://doi.org/10.15200\%2Fwinn.144503.33399.

Hontas Farmer and. A Simple Calculation That Shows Black Hole Firewalls Exist and How Hot They Are., b. URL https://doi.org/10.15200\%2Fwinn.146254.45957.

Hontas Farmer and. Fundamentals of Relativization. The Winnower, 2014a. doi: 10.15200/winn.141487. 76774. URL https://doi.org/10.15200\%2Fwinn.141487.76774.

Hontas Farmer and. Quantum gravity by relativization of Quantum Field Theory. The Winnower, 2014b. doi: 10.15200/winn.140751.17561. URL https://doi.org/10.15200\%2Fwinn.140751.17561.

Hontas Farmer and. Fundamentals of Relativization II with Computational Analyses. The Winnower, 2015. doi: 10.15200/winn.142574.40936. URL https://doi.org/10.15200\%2Fwinn.142574. 40936.

ATLAS Collaboration. Search for New Phenomena in a Lepton Plus High Jet Multiplicity Final State with the Atlas Experiment Using $\sqrt{S}=13$ Tev Proton-Proton Collision Data. 2017. ArXiv e-prints, apr 2017.

H. Farmer. Quantum Gravity, May Not Be The Right Question. 2017. In APS April Meeting Abstracts, January 2017.

Search for supersymmetry using hadronic top quark tagging in $13 \mathrm{TeV}$ pp collisions. Technical Report Cms-Pas-Sus-16-050, Cern, Geneva. 2017. URL http://cds.cern.ch/record/2262651.

L. Susskind. Dear Qubitzers, GR=QM. ArXiv e-prints, aug 2017. 